Methods The NETS is a commercial dataset providing retail business information in the United States. NYC data were acquired for the period 1990-2010. Businesses were grouped into researcher-defined categories based on Standard Industrial Classification codes and other fields such as business name. All businesses were re-geocoded to ensure accurate localisation. We defined access to BMI-unhealthy businesses (characterised as selling calorie-dense foods such as pizza and pastries) as the total number of BMI-unhealthy businesses present in each NYC census tract $(n=2,167)$ in January of each year. We conducted LCGA in Mplus to identify census tracts with similar trajectories of BMI-unhealthy businesses. We used model fit statistics and interpretability to determine the number of classes. Using the final models, we assigned census tracts to latent classes. We predicted class membership with socio-demographic variables from the Census (population size, income, and ethnic composition) using multinomial logistic regressions and reported predicted probabilities with 95\% CI. Sensitivity analyses were undertaken.

Results The final models include 5 and 10 latent classes, respectively. The 5 -class solution indicates an overall increase in the number of BMI-unhealthy businesses over time and shows a pattern of fanning out: the higher the value in 1990, the greater the increase over time. Classes are associated with 1990 population size, income, proportion of Black residents (all $\mathrm{p}<0.001)$, proportion of Hispanic residents $(\mathrm{p}=0.033)$, and 1990-2010 change in population size and income $(\mathrm{p}<0.001)$. The 10-class solution identifies two pairs of classes with similar 1990 values, but different trajectories. Differences in those trajectories are associated with population size and ethnic composition $(\mathrm{p}<0.001)$.

Conclusion This study illustrates how LCGA contributes to the understanding of long-term exposure to the obesogenic environment. The technique can easily be applied to other aspects of the neighbourhood and to other geographies. When linked with health data, identified latent classes can be used to assess how longitudinal exposure to changing neighbourhoods affects health.

\section{P28 ASSOCIATION BETWEEN INDOOR TEMPERATURE AND GENERAL HEALTH IN ENGLISH ADULTS: A CROSS- SECTIONAL STUDY}

1,2 J Sutton-Klein*, 'A Moody, J Mindell. 'Institute of Epidemiology and Health, University
College London, London, UK; ${ }^{2}$ The Medical School, University of Sheffield, Sheffield, UK

10.1136/jech-2017-SSMAbstracts. 130

Background While the association between indoor temperature and health has been indirectly investigated using variables including self-reported cold homes and the presence of insulation as proxies for temperature, research using indoor temperature directly has been lacking. We hypothesised that lower indoor temperatures would be associated with increased odds of poor general health, which would be partially explained by socioeconomic and housing variables.

Methods We used data from the Health Survey for England collected between 2003 and 2014. The analytical sample consisted of 74735 participants aged over 16. Indoor temperature was approximated using a one-off reading of indoor air temperature in participants' homes. Self-reported general health was used for the main outcome, dichotomised into very good/good/fair vs. bad/very bad.
Using Stata, logistic regression models were built for the data, using maximum likelihood estimation. The complex survey design and weights were taken into account in the analysis. The influence of socioeconomic and housing factors was assessed by adjusting for these variables in the models.

Results The mean indoor temperature was $20.6^{\circ} \mathrm{C}$ (SE 0.09), which varied seasonally and regionally. Warmer indoor temperatures were associated with greater odds of poor general health. The age and sex-adjusted odds ratio (OR) of poor health for each degree increase in indoor temperature was 1.01 (95\% CI 1.01-1.02 P<0.01). Lower social class and lower education level were associated with warmer temperatures and poorer health. The OR decreased after adjusting for these confounding variables. Higher income was associated with warmer temperatures and fairer health. After adjusting for both the suppressor and confounding variables, the OR for poor health for each degree increase in temperature has a statistically significant increase by $19 \%$ to 1.02 (95\% CI $1.01-$ $1.03 \mathrm{P}<0.01)$.

Conclusion Our analysis unexpectedly showed an association between high indoor temperature and poor health. The relationship between socioeconomic factors and indoor temperature was complicated, with different socioeconomic variables having different directions of association with indoor temperature. Further research is needed to understand the temporality and direction of the association. The research is timely given the potential for data from increasingly prevalent smart thermostats.

\section{P29 RACIAL INEQUALITIES IN DENTAL SERVICE UTILISATION AMONGST MIDDLE-AGED BRAZILIAN ADULTS}

HM Constante*, G Tsakos, RG Watt. Department of Epidemiology and Public Health, University College London, London, UK

\subsection{6/jech-2017-SSMAbstracts. 131}

Background Dental service utilisation is important as it helps to alleviate pain and suffering, and access to dental treatment. International evidence has shown that racial/ethnic minority groups are less likely to use dental services compared to the White majority population. In Brazil, where the concept of colour/race is used to classify the population, recent research has assessed colour/race inequalities in dental service use amongst the elderly population, but evidence is scarce for other age groups. Therefore, the overall aim of this research is to investigate the differences between colour/race groups (White versus Pardo and Black) in the use of dental service in a national sample of 35 to 44 year-old adults in Brazil taking into consideration the role of individual-level characteristics. Methods Data from 7902 adults aged 35 to 44 years from the Brazilian National Oral Health Survey (SB Brazil 2010) was used in this study. The survey collected data using an interviewer-administrated questionnaire and clinical examinations. Dependent variables were the time since last dental visit (less than a year, or a year or more), reason for the last dental visit (prevention/check-up, pain or extraction, or treatment), and type of service used (public, or private). The main exploratory variable was self-reported colour/race (White, Pardo, or Black) measured according to the Brazilian Institute of Geography and Statistics (IBGE). Covariates were sex, level of education, family income, satisfaction with teeth/mouth, self-reported need for treatment, self-reported dental pain in 
the last six months, presence of decayed teeth, filled teeth, and missing teeth. Binary and multinomial logistic regression analyses were conducted weighted for complex sample design. Results No association between colour/race and time since last dental visit on the fully fitted model was observed. For the comparison between visits due to preventive/check-up and pain or extraction, the association remained significant only for Pardos compared to Whites after the adjustments (RRR 1.63 ; 95\% CI 1.11-2.38). In contrast, there was no association between colour/race and visiting the dentist due to treatment. Results from the adjusted model showed statistical differences only for Blacks, and this group was 1.48 times (95\% CI 1.10-1.97) more likely to visit the public dental service compared to Whites.

Conclusion Racial inequalities in dental service utilisation were evident for middle-aged adults in Brazil. For some outcomes, the study showed that colour/race inequalities were not fully explained by the covariates investigated. Further multilevel analysis will be conducted to better understand these inequalities.

\section{P30 DOES THE QUALITY OF SOCIAL RELATIONSHIPS BUFFER THE ASSOCIATION OF SOCIAL DISADVANTAGE WITH ALLOSTATIC LOAD? AN ANALYSIS OF ADULTS FROM THE UK HOUSEHOLD LONGITUDINAL STUDY}

${ }^{1} \mathrm{P}$ Rouxel ${ }^{*},{ }^{2} \mathrm{~T}$ Chandola, ${ }^{3} \mathrm{M}$ Benzeval. ${ }^{1}$ Social Science, UCL Institute of Education, London, UK; ${ }^{2}$ Institute for Social and Economic Research, University of Essex, Colchester, UKi ${ }^{3} \mathrm{CMIST}$ and Social Statistics, University of Manchester, Manchester, UK

\subsection{6/jech-2017-SSMAbstracts.132}

Background Positive support from social relationships is associated with better health, although it remains debatable whether positive social support buffers against the negative effects of social disadvantage on health. Moreover, few studies have considered both positive and negative relationship features from different networks (partner, relatives, and friends) and their association with allostatic load, a multisystem physiological dysregulation index, and none have examined whether the association of social disadvantage with higher levels of allostatic load reduces among those with more positive social support (the stress buffering role of positive social support).

Methods This study examined data from 7928 adults from the UK Household Longitudinal Study (wave 2-2010/11). Positive and negative social relationships were assessed within networks (partner, relatives, friends). Allostatic load (range 0-10) was measured by summing eleven risk scores across neuroendocrine, immune, metabolic, cardiovascular and anthropometric systems. We used negative binomial regression models to examine the association of positive social support and negative social interactions with allostatic load, controlling for socioeconomic/demographic, health, behavioural and personality factors.

Results Positive support from all networks was associated with lower allostatic load. Similarly, negative interactions from all networks were associated with higher allostatic load. However, after adjusting for all covariates, only positive support from partners and relatives, and negative interactions with friends were associated with allostatic load. Men with degree qualifications and supportive partners had lower allostatic load (2.3; 95\% CI: 2.2-2.5) than highly educated men with low partner support $(2.7$; 95\% CI: 2.4-3.0); positive support did not buffer the association of low education with allostatic load.
Unemployed adults with supportive partners had lower allostatic load $(2.4 ; 95 \% \mathrm{CI}: 1.9-2.8)$ compared to unemployed adults with low partner support (3.2; 95\% CI: 2.4-4.0).

Conclusion Positive social support from partners appears to buffer the effect of unemployment on allostatic load. However, contrary to the stress buffering hypothesis, highly educated men appear to benefit more from supportive partners than men and women with no qualifications.

\section{P31 ACCESS TO PRIMARY CARE FOR SOCIO-ECONOMICALLY DISADVANTAGED OLDER PEOPLE IN RURAL AREAS}

${ }^{1}$ JA Ford*, ${ }^{1}$ AP Jones, ${ }^{2} \mathrm{G}$ Wong, ${ }^{1} \mathrm{~A}$ Clark, ${ }^{1} \mathrm{~T}$ Porter, ${ }^{1} \mathrm{~N}$ Steel. ${ }^{1}$ Norwich Medical School, University of East Anglia, Norwich, UK; ${ }^{2}$ Nuffield Department of Primary Care, University of Oxford, Oxford, UK

\subsection{6/jech-2017-SSMAbstracts.133}

Background Our previous realist review and qualitative research found that socio-economically disadvantaged older people in rural areas face personal, community and health care barriers to obtaining an appointment in primary care. We described these barriers with context-mechanism-outcome (CMO) configurations. Example contexts were lifelong poverty, education, previous healthcare experience, transport, mechanisms were health literacy, assertiveness and convenience, and the outcome was obtaining an appointment. We aimed to explore these CMO configurations using Structural Equation Modelling (SEM) in a national linked dataset.

Methods Individual patient data from Wave 6 of the English Longitudinal Study of Ageing (ELSA) was linked with practice data from the GP Patient Survey (GPPS). Participants in the lowest socio-economic group and living in a rural area were included. Variables from ELSA and GPPS were available for nine complete CMOs. Confirmatory factor analysis was used to generate seven latent variables for unobserved concepts such as lifelong poverty and previous healthcare experience. CMOs were analysed in one overall SEM with multiple mediation paths; contexts were treated as the exposure, mechanisms as the mediator, and the common outcome was ability to obtain an appointment. Stata and MPlus was used to estimate standardised coefficients and 95\% confidence intervals using robust maximum likelihood.

Results 276 patients from 178 different GP surgeries were included. We found statistically significant direct or indirect effects for two of the nine CMOs. The ease of getting through to the surgery was statistically significantly associated with being able to get an appointment ( $\beta$ 0.52, 0.42 to 0.61$)$ with an indirect, statistically significantly, mediated effect through convenience ( $\beta 0.14,0.07$ to 0.21 ). Health care experience was not directly associated with getting an appointment $(\beta-0.04,-0.19$ to 0.12$)$, but a statistically significant indirect effect through convenience existed ( $\beta$ 0.10, 0.04 to 0.16 ). Model fit showed mixed results (RMSEA 0.05, CFI 0.923, TLI 0.901) Analysis was limited because there were not data for all theoretical concepts. Furthermore, continuous variables were only included to obtain adequate model fit.

Conclusion We found that obtaining an appointment was both directly associated with the ease of the booking system, and mediated through the mechanism of perceived convenience. We also found a mediated effect from previous health care experience to obtaining an appointment through perceived convenience. 